INFLAMMATORY BOWEL DISEASE

\title{
CARD15/NOD2 gene variants are associated with familially occurring and complicated forms of Crohn's disease
}

\author{
T Heliö, L Halme, M Lappalainen, H Fodstad, P Paavola-Sakki, U Turunen, M Färkkilä, \\ T Krusius, K Kontula
}

Gut 2003;52:558-562

See end of article for authors' affiliations

Correspondence to: Dr T Heliö, Helsinki University Hospital Department of Medicine, Haartmaninkatu 4, 00290 Helsinki, Finland; tiina.helio@hus.fi.

Accepted for publication 15 October 2002

\begin{abstract}
Background: Variants of the caspase activating recruitment domain 15/nucleotide oligomerisation domain 2 (CARD15/NOD2) gene have been associated with susceptibility to Crohn's disease (CD). Aim: Our aim was to evaluate the allele frequencies of the CARD 15 variants R702W, G908R, and $1007 \mathrm{fs}$ in Finnish inflammatory bowel disease (IBD) patients and to search for possible associations between CARD 15 variants and occurrence of familial forms of IBD or complicated forms of CD.

Patients and methods: We investigated 198 sporadic CD patients, 46 probands with familial CD, 27 CD probands from mixed IBD families, 99 unrelated patients with ulcerative colitis (UC), and 300 control individuals for the occurrence of the CARD15 gene variants R702W, G908R, and $1007 \mathrm{fs}$.

Results: In CD patients, the allele frequencies for the rare variants of these polymorphisms were $3.3 \%$, $0.6 \%$, and $4.8 \%$ (total $8.7 \%$ ), and the corresponding frequencies in healthy controls were $1.8 \%, 0 \%$, and $1.7 \%$ (total $3.5 \%)(8.7 \% \vee 3.5 \% ; p<0.01)$. In UC patients allele frequencies were comparable with those in controls. The frequency of the $1007 \mathrm{fs}$ polymorphism variant allele was significantly higher among all CD patients than in controls $(4.8 \%$ v $1.7 \% ; p<0.01)$ but there was no significant difference in allele frequencies between the CD and UC groups. The $1007 \mathrm{fs}$ allele frequency was higher in familial $C D$ than in non-familial cases with $C D(10.9 \%$ v $3.5 \% ; p<0.01)$. There were no significant differences in the allele frequencies of the R702W and G908R polymorphisms between CD patients, UC patients, and controls. We found that $15.5 \%$ of CD patients, $9.1 \%$ of UC patients, and $6.7 \%$ of controls carried at least one of the CARD 15 variants. In CD patients carrying at least one of the three NOD2 variants, the ileum was affected more often than in non-carrier CD patients $190 \% \vee 73 \%$; $\mathrm{p}<0.05)$, they had stricturing or penetrating disease more often than non-carriers $(88 \% \vee 56 \%$; $p<0.011$, and they had an increased need for bowel surgery.

Conclusions: The frequency of NOD2 gene variants was lower in genetically homogenous Finns than in other populations. The $1007 \mathrm{fs}$ variant was associated with CD. The occurrence of CARD 15 variants predicted ileal location as well as stricturing and penetrating forms of $C D$.
\end{abstract}

rohn's disease (CD) and ulcerative colitis (UC) are relatively common major forms of chronic inflammatory bowel diseases (IBD). Both genetic and environmental factors have been suggested to predispose to CD and UC. Among Finns, affected first degree relatives were recently discovered in $15.6 \%$ of patients with CD and in $13.8 \%$ of patients with UC, values comparable with those reported elsewhere in Europe. ${ }^{1}$ A number of linkage studies carried out in separate populations have confirmed the genetic linkage of CD to chromosome 16. ${ }^{2-10}$ Hugot and colleagues ${ }^{11}$ and Ogura and colleagues ${ }^{12}$ have independently unravelled the $\mathrm{CD}$ associated nucleotide oligomerisation domain 2 (NOD2) gene, located on chromosome $16 \mathrm{q} 12$ within the area of strongest linkage to $\mathrm{CD}$. NOD2, recently renamed as caspase activating recruitment domain 15 (CARD 15), appears to belong to a family of NODI/ apoptotic protease activating factor 1 (Apaf-1) related proteins which regulate apoptosis and some of which activate nuclear factor $\mathrm{KB}(\mathrm{Nf} \kappa \mathrm{B}) .^{13}{ }^{14}$ Unlike NODl and Apaf-1, CARD15 is mainly expressed in monocytes, but not in lymphocytes, normal colon, or the small intestine. The exact function of CARD15 remains unknown but it seems to play a role both in intracellular recognition of lipopolysaccharides (LPS) typical of Gram negative bacteria and subsequent activation of NFKB. ${ }^{15}$

More than 60 sequence variants have been identified in the CARD15 gene, with three main variants (R702W, G908R, and 1007fs) proposed to be associated with susceptibility to
$\mathrm{CD}^{11}{ }^{12}{ }^{16-19}$ One of these ( $1007 \mathrm{fs}$ ), which shows the most consistent association with $\mathrm{CD}$, has also been reported to result in phenotypic consequences. Insertion of an extra cytosine molecule causes a translational frameshift creating a premature stop codon in the 10th leucine rich repeat (LRR) of CARD15, and this alteration has been shown to decrease NFKB activation by LPS. ${ }^{12}$

The present study was undertaken to evaluate the occurrence of the CARD15 variants R702W, G908R, and 1007fs in Finnish IBD patients. Our particular aim was to search for associations between hetero- or homozygosity for the CARDI5 variants, familial forms of IBD, and complicated forms of CD.

\section{MATERIALS AND METHODS \\ Participants}

Consecutive Finnish patients with familial and sporadic IBD were recruited from the capital city of Helsinki by investigators at the Helsinki University Hospital and Maria Hospital. In addition, IBD families were recruited by collaborators from

Abbreviations: Apaf-1, apoptotic protease activating factor 1 ; CARD caspase activating recruitment domain; CD, Crohn's disease; IBD, inflammatory bowel disease; LPS, lipopolysaccharide; LRR, leucine rich repeats; NFKB, nuclear factor $\kappa B ; N O D$, nucleotide oligomerisation domain; PCR, polymerase chain reaction; UC, ulcerative colitis. 
University Hospitals in Turku, Tampere, Kuopio and Oulu, as described previously. ${ }^{120}$ Patients and their affected first degree relatives completed a questionnaire concerning the history and details of their disease as well as the number of affected and non-affected relatives. Subjects with familial CD had at least one first degree relative with IBD whereas subjects with sporadic CD did not. In pure familial CD, all patients in the same family had CD. In mixed families, the proband had CD and at least one first degree relative had UC or indeterminate colitis. Details on the course of the disease in participants with IBD were obtained from patient records. Standard endoscopic and histological criteria were used to establish the diagnosis. ${ }^{21}$ The Vienna classification was used to grade $\mathrm{CD}^{22}$ For location of disease, we used the largest extent of the disease according to $x$-ray and endoscopy protocols as well as surgical reports. The behaviour of the disease was classified as penetrating if there were intra-abdominal or perianal fistulas at any time during the course of the disease, even if there were coexisting strictures.

In total, we studied DNA samples from 198 patients with sporadic $C D, 46$ probands with familial $C D, 27$ unrelated probands with CD from mixed IBD families, and 99 randomly chosen unrelated patients with familial and sporadic UC. Informed written consent was obtained from all patients. DNA samples from 300 healthy blood donors were obtained through the Finnish Red Cross Blood Service (Helsinki, Finland). Consent obtained for the use of blood from these donors precluded collection of any clinical data.

This study was approved by the ethics review committees of each of the participating centres.

\section{Analysis of CARD15 variants}

Genomic DNA was extracted from venous blood samples by standard procedures using digestion with proteinase $\mathrm{K}$ followed by phenol/chloroform extraction. Each CARD15 variant was assayed using initial amplification of the DNA sample by polymerase chain reaction (PCR) and subsequent analysis of the PCR products by restriction enzyme cleavage and gel electrophoresis on $12 \%$ polyacrylamide (R702W) or 3-4\% agarose (G908R and 1007fs).

For assay of the R702W mutation, the forward primer was 5'-AGATCACAGCAGCCTTCCTG-3' and the reverse primer was $5^{\prime}$ - CACGCTCTTGGCCTCACC-3'. The PCR product ( $185 \mathrm{bp}$ in size) was digested at $37^{\circ} \mathrm{C}$ for 16 hours with $2 \mathrm{U}$ of MspI, resulting in the following fragments: $20,35,54$, and $76 \mathrm{bp}$ in R702R homozygotes; 20, 35, 54, 76, and 130 bp in R702W heterozygotes; and 20, 35, and $130 \mathrm{bp}$ in W702W homozygotes.

For assay of the G908R mutation, 5' CTCTTTTGGCCTTTTCAGATTCTG-3' was used as the forward primer and 5'-CAGCTCCTCCCTCTTCACCT- $3^{\prime}$ as the reverse primer. The PCR product size from these primers is $163 \mathrm{bp}$ After digestion for 16 hours at $37^{\circ} \mathrm{C}$ with $2 \mathrm{U}$ of Hha I, the following fragments were obtained: 163 bp in G908G homozygotes; 27, 136, and 163 in G908R heterozygotes; and 27 and 136 bp in R908R homozygotes.

In order to detect the 1007fs mutation, PCR was carried out using the forward primer 5'. GGCAGAAGCCCTCCTGCAGGGCC-3' and the reverse primer $5^{\prime}$ - CCTCAAAATTCTGCCATTCC- $3^{\prime}$ resulting in an amplified fragment of $151 \mathrm{bp}$ in size. After digestion for 16 hours at $37^{\circ} \mathrm{C}$ with $2 \mathrm{U}$ of ApaI, the following panel was obtained (leucine represents the codon 1007 in the wild-type allele): 151 bp for Leu 1007Leu homozygotes; 20, 131, and 151 bp in Leu 1007Pro heterozygotes; and 20 and 131 bp in Prol007Pro homozygotes.

\section{Statistical analyses}

Frequencies between patient and control groups were tested using Pearson's $\chi^{2}$ statistics or Fishers's exact test. The Student's $t$ test for unpaired samples was used to compare continuous data between two groups of patients. Bonferroni's correction was used to take into account the number of hypotheses tested.

\section{RESULTS}

Allele frequencies in patients and controls

The CARD15 gene variants R702W, G908R, and 1007fs were determined in 198 unrelated sporadic CD patients, 46 unrelated probands with familial CD, 27 unrelated probands with CD from mixed IBD families, 99 randomly chosen unrelated familial and sporadic UC patients, and in 300 healthy blood donors. The CARD 15 allele and genotype frequencies are summarised in table 1 . The allele frequency of the $1007 \mathrm{fs}$ mutation in familial CD cases was $10.9 \%$, in sporadic CD patients $3.5 \%$, in CD patients from mixed families $3.7 \%$, in UC patients $3.0 \%$, and in controls $1.7 \%$. The allele frequency of the 1007 fs variant was significantly higher among all CD patients than in controls $(4.8 \% v 1.7 \% ; \mathrm{p}<0.01)$, and the corresponding frequency in familial CD cases was higher than in sporadic CD cases $(10.9 \% \vee 3.5 \% ; \mathrm{p}<0.01)$. The frequencies of the R702W and G908R variants were relatively low in all patient categories and in controls, with no significant differences between the groups (table 1): $15.5 \%$ of CD patients, $9.1 \%$ of UC patients, and $6.7 \%$ of control subjects carried at least one of the CARD15 polymorphisms. The difference in allele frequencies between the CD group and controls, but not between other groups, was significant $(\mathrm{p}<0.01)$.

One of 198 patients with sporadic CD and two of 46 patients with familial CD were homozygous for the 1007fs mutation. Other CD patients carrying these three variants were also heterozygous (table 1). A total of nine (9.1\%) of 99 UC patients were heterozygous carriers of one of the three CARD15 variants (table 1). There were two combined heterozygotes in relation to the R702W and G908R variants among CD patients (one sporadic and one familial CD case), and one compound heterozygote in relation to $\mathrm{R} 702 \mathrm{~W}$ and 100fs polymorphisms among controls.

\section{CARD15 alleles and clinical phenotypes}

When median ages at diagnosis in those CD patients who were hetero- or homozygous for any of the rare CARD15 variants $(\mathrm{n}=42)$ and in those homozygous for the wild-type CARD15 allele $(n=229)$ were compared, no significant differences were noted (table 2). In contrast, median disease duration was approximately twice as high in the former than in the latter group (table 2). Based on the Vienna classification, CD patients with at least one of the rare CARD15 variants had more often the ileum affected $(90 \% v 73 \% ; \mathrm{p}<0.05)$ and more often a stricturing or penetrating form of the disease $(88 \% \mathrm{v}$ $56 \% ; \mathrm{p}<0.01$ ) compared with non-carrier CD patients (table $3)$. In addition, carriers of the CARD15 variant alleles had undergone bowel surgery more often than non-carriers (67\% $v 46 \% ; \mathrm{p}<0.05$ ) (table 2 ). There were no significant differences in the use of 5-ASA, metronidazole, steroids, or immunosuppressives (azathioprine, methotrexate, or cyclosporine) between CD patients who were carriers of any of the three CARD15 variants investigated compared with non-carriers (table 3).

When UC patients were classified into carriers of any of these CARD15 polymorphisms and non-carriers, no significant differences were observed with regard to mean age, median age at diagnosis, median duration, need for medication, or need for surgery (not shown). Of the CD patients, $48 \%$ were smokers and $17 \%$ were ex- or casual smokers, while the corresponding frequencies among UC patients were 13\% and $35 \%(\mathrm{p}<0.01)$.

\section{Characteristics of patients and their affected siblings homozygous for $1007 \mathrm{fs}$}

Both of the two familial CD patients homozygous for the CARD15 1007fs mutation had one affected sibling who was 
Table 1 Carrier and allele frequencies of the CARD 15 gene variants R702W, G908R, and 1007fs in patients with Crohn's disease (CD) or ulcerative colitis (UC) and in controls

\begin{tabular}{|c|c|c|c|c|}
\hline \multirow[b]{2}{*}{ Group } & \multicolumn{3}{|c|}{ CARD 15 genotype frequency } & \multirow[b]{2}{*}{$\begin{array}{l}\text { CARD } 15 \text { variant } \\
\text { allele frequency } \\
\text { (\%) }\end{array}$} \\
\hline & $\begin{array}{l}\text { Homozygotes } \\
(n /(\%))\end{array}$ & $\begin{array}{l}\text { Heterozygotes } \\
(\mathrm{n} /(\%))\end{array}$ & $\begin{array}{l}\mathrm{Wt} / \mathrm{Wt} \\
(\mathrm{n} /(\%))\end{array}$ & \\
\hline \multicolumn{5}{|c|}{ CD sporadic $(n=198)$} \\
\hline $\mathrm{R} 702 \mathrm{~W}$ & $0 /(0.0)$ & $13 /(6.6)$ & $185 /(93.4)$ & 3.3 \\
\hline G908R & $0 /(0.0)$ & $2 /(1.0)$ & 196/(99.0) & 0.5 \\
\hline $1007 \mathrm{fs}$ & $1 /(0.5)$ & $12 /(6.1)$ & $185 /(93.4)$ & 3.5 \\
\hline \multicolumn{5}{|c|}{ CD familial $(n=46)$} \\
\hline R702W & $0 /(0.0)$ & $3 /(6.5)$ & $43 /(93.5)$ & 3.3 \\
\hline G908R & $0 /(0.0)$ & $1 /(2.2)$ & $45 /(97.8)$ & 1.1 \\
\hline $1007 \mathrm{fs}$ & $2 /(4.4)$ & $6 /(13.0)$ & $38 /(82.6)$ & $10.9 *$ \\
\hline \multicolumn{5}{|c|}{$C D$ mixed families $(n=27)$} \\
\hline R702W & $0 /(0.0)$ & $2 /(7.4)$ & $25 /(92.6)$ & 3.7 \\
\hline G908R & $0 /(0.0)$ & $0 /(0.0)$ & $27 /(100.0)$ & 0.0 \\
\hline $1007 \mathrm{fs}$ & $0 /(0.0)$ & $2 /(7.4)$ & $25 /(92.6)$ & 3.7 \\
\hline \multicolumn{5}{|c|}{ CD all $(n=271)$} \\
\hline R702W & $0 /(0.0)$ & $18 /(6.6)$ & $253 /(93.4)$ & 3.3 \\
\hline G908R & $0 /(0.0)$ & $3 /(1.1)$ & $268 /(98.9)$ & 0.6 \\
\hline $1007 \mathrm{fs}$ & $3 /(1.1)$ & $20 /(7.4)$ & $248 /(91.5)$ & $4.8+$ \\
\hline \multicolumn{5}{|l|}{ UC (n=99) } \\
\hline R702W & $0 /(0.0)$ & $3 /(3.0)$ & $96 /(97.0)$ & 1.5 \\
\hline G908R & $0 /(0.0)$ & $0 /(0.0)$ & $99 /(100.0)$ & 0.0 \\
\hline $1007 \mathrm{fs}$ & $0 /(0.0)$ & $6 /(6.1)$ & $93 /(94.0)$ & 3.0 \\
\hline \multicolumn{5}{|c|}{ Controls $(n=300)$} \\
\hline R702W & $0 /(0.0)$ & $11 /(3.7)$ & $289 /(96.3)$ & 1.8 \\
\hline G908R & $0 /(0.0)$ & $0 /(0.0)$ & $300 /(100.0)$ & 0.0 \\
\hline $1007 f_{s}$ & $0 /(0.0)$ & $10 /(3.3)$ & $290 /(96.7)$ & 1.7 \\
\hline
\end{tabular}

* $1007 \mathrm{fs}$ allele frequencies, $C D$ familial versus $C D$ sporadic: $p<0.01$.

$\dagger 1007 \mathrm{fs}$ allele frequencies, $C D$ all versus controls: $p<0.01$.

$\mathrm{Wt}$, wild-type allele.

Table 2 Characteristics of patients with Crohn's disease (CD) according to CARD15 gene status

\begin{tabular}{|c|c|c|c|c|c|}
\hline \multirow{3}{*}{$\begin{array}{l}\text { Variable } \\
\text { Familial }\end{array}$} & \multicolumn{4}{|c|}{ CARD 15 status } & \multirow{3}{*}{$\frac{p \text { Value }}{\text { NS }}$} \\
\hline & \multicolumn{2}{|c|}{$\begin{array}{l}\text { Wt/Wt homozygotes } \\
(n=229)\end{array}$} & \multicolumn{2}{|c|}{$\begin{array}{l}\text { Carriers of variants } \\
(n=42)\end{array}$} & \\
\hline & 58 & $(25 \%)$ & 15 & $(36 \%)$ & \\
\hline Pure disease/CD ( $\mathrm{n}=46)$ & 35 & & 11 & & \\
\hline Mixed $(n=27)$ & 23 & & 4 & & \\
\hline Men $(\%)$ & 99 & $(43 \%)$ & 20 & $(48 \%)$ & NS \\
\hline Median (range) age at diagnosis (y) & 27.5 & $5(9-65)$ & 26.8 & $(7-69)$ & NS \\
\hline Median (range) duration (y) & & $(0-46)$ & 12.3 & $(0.3-29)$ & $<0.01$ \\
\hline \multicolumn{6}{|l|}{ Medication } \\
\hline 5-ASA & 222 & (97\%) & 38 & $(90 \%)$ & \\
\hline Metronidazole & 144 & (63\%) & 24 & (57\%) & NS \\
\hline Steroids & 172 & (75\%) & 31 & (74\%) & \\
\hline Immunosuppressives $\dagger$ & 91 & $(40 \%)$ & 15 & (36\%) & \\
\hline Bowel surgery (\%) & 105 & $(46 \%)$ & 28 & (67\%) & $<0.05$ \\
\hline \multicolumn{6}{|l|}{ Indication for surgery } \\
\hline Emergency & 5 & & 3 & & \\
\hline Complication & 85 & & 23 & & \\
\hline Chronic active disease & 15 & & 1 & & \\
\hline Dysplasia/carcinoma & 0 & & 1 & & \\
\hline \multicolumn{6}{|l|}{ Smoking } \\
\hline No & 73 & $(32 \%)$ & 16 & $(38 \%)$ & \\
\hline Ex or casual & 36 & $(16 \%)$ & 7 & (17\%) & NS \\
\hline Yes & 106 & $(46 \%)$ & 17 & $(40 \%)$ & \\
\hline
\end{tabular}

also found to be homozygous for the 1007fs polymorphism. As a group, these four CD patients homozygous for 1007fs shared some phenotypic characteristics: age at diagnosis was 20 years or less, only the ileum was affected, and CD showed a stricturing course. The single sporadic CD patient who was homozygous for the 1007fs allele differed from these two sibling pairs in that, in addition to the ileum, the right colon was also affected. His disease was diagnosed at the age of 13 years, stricture formation complicated the disease, and the patient had undergone three bowel resections.

\section{DISCUSSION}

Since 2001, several independent studies have demonstrated that the NOD2/CARD15 gene variants contribute to a significant extent to the risk of Crohn's disease. ${ }^{11}{ }^{12}{ }^{16-19}$ The allele frequency of the insertion mutation 1007fs, predicted to result in 
Table 3 Vienna classification of patients with Crohn's disease according to CARD 15 gene status

\begin{tabular}{|c|c|c|c|}
\hline \multirow[b]{2}{*}{ Variable } & \multicolumn{2}{|c|}{ CARD 15 gene status } & \multirow[b]{2}{*}{$\mathrm{p}$ Value } \\
\hline & $\begin{array}{l}\text { Wt/Wt } \\
\text { homozygotes } \\
(n=229)\end{array}$ & $\begin{array}{l}\text { Carriers of } \\
\text { variants } \\
(n=42)\end{array}$ & \\
\hline \multicolumn{4}{|l|}{ Age at onset } \\
\hline$<40 y$ & 183 & 36 & NS \\
\hline$\geqslant 40 y$ & 46 & 6 & \\
\hline \multicolumn{4}{|l|}{ Location } \\
\hline Colon & 62 & 4 & \\
\hline lleum & 49 & 15 & $<0.05$ \\
\hline lleocolon & 112 & 19 & \\
\hline Upper GI tract & 6 & 4 & \\
\hline \multicolumn{4}{|l|}{ Behaviour } \\
\hline Inflammatory & 101 & 6 & \\
\hline Stricturing & 78 & 20 & $<0.01$ \\
\hline Penetrating & 50 & 17 & \\
\hline
\end{tabular}

a truncated CARD15 protein, was found to be $8-16 \%$ in CD patients but only $2-4 \%$ in control populations from the USA ${ }^{12}$ or Europe. ${ }^{11}{ }^{16-18}$ Two other mutations (R702W and G908R) have also shown a significant relation to the risk of $\mathrm{CD}^{11}{ }^{17-19}$ while there was no significant association between CARD15 gene variants and the risk of UC. Hampe et al estimated that the relative risk of CD in 1007fs heterozygotes was 2.6 and in homozygotes $42.6 .{ }^{16}$ Cuthbert and colleagues ${ }^{18}$ reported a relative CD risk of 3.0 for individuals heterozygous for any of the three CARD15 gene variants and a value of 23.4 for homozygotes or compound heterozygotes. According to Hugot et al, the corresponding relative risks were 3,38 , and 44 , respectively. ${ }^{1 .}$

Our material of familial CD was limited as the whole population comprised approximately 5 million inhabitants and secondly, there is no national IBD register, which makes it more difficult to recruit patients. In our material, the frequencies of all of the CARD15 variants investigated were low in comparison with many other Western populations. ${ }^{11}{ }^{12} 16-19$ Recently, Inoue et al have reported lack of common NOD2 variants in Japanese subjects with $\mathrm{CD} .{ }^{23}$ Our data confirm the association between the CARD15 1007fs variant allele and CD, although there was no difference in allele frequencies between CD and UC groups. This association may not be as strong in the Finnish population as in other Caucasian populations studied to date. There were no significant differences in the allele frequencies of the two other CARD15 gene variants investigated (R702W and G908R) between patients with CD and UC or controls. The CARD15 1007fs mutation was more prevalent among probands with familial disease compared with sporadic cases. The studies by Hugot et al and Ogura et al, showing an association between CARD 15 mutations and CD, also comprised familial cases. ${ }^{11}{ }^{12}$ As suggested by the observations by Inoue et al, there may be other disease causing polymorphisms of the CARD15 gene, or entirely other genes, which predispose to $C D$. The search for other clinically significant CARD15 variants among the Finns is warranted.

The Finns derive from a small relatively isolated founder population in which the population growth was rapid and breeding units were small. ${ }^{24}$ The Finnish population has been extensively and successfully investigated for several rare monogenic diseases. ${ }^{25} \mathrm{~A}$ few more common diseases, such as familial hypercholesterolaemia or long QT syndrome, are caused by founder mutations typical of Finns. ${ }^{26}{ }^{27}$ Recently, the properties of isolated populations in mapping variants underlying complex diseases have been evaluated..$^{24} 2829$ The fate of alleles with a frequency of $1 \%$ in a rapidly growing population, allowed to mate randomly, was simulated by computer and after 20 generations the frequency of the most common allele was $6 \%$ whereas some alleles were lost. ${ }^{24}$ This means that ran- dom genetic drift has a strong influence on allele frequencies in small fast expanding populations: rare alleles may get lost or survive at unusually high frequencies..$^{24}$ If a disease gene has several mutations, some may have been lost and some major mutations preserved.

We have previously shown that the percentage of familial IBD among Finnish patients is comparable with that in other European populations. ${ }^{1}$ No remarkable clinical differences were observed between patients with familial or sporadic disease. ${ }^{1}$ In the present study, a carrier status for any of the three CARD15 variants, and especially for the 1007fs mutation, predicted ileal localisation of CD as well as liability to complications of $\mathrm{CD}$, such as strictures, fistulations, and need for surgical revisions. Very recently, similar findings were reported by other investigators. ${ }^{16-19}$ An increased frequency of the CARD15 mutations in ileal specific disease has been reported in several independent studies, ${ }^{17-19}$ and Hampe et al also reported an association between CARD15 variants with right colonic disease in comparison with left colon specific disease. ${ }^{30}$ In our study, the rare homozygous patients had a form of CD with an early onset and a complicated course, a finding strikingly similar to that of Lesage and colleagues ${ }^{19}$ observed in a French population. In the present series the median duration of disease was twice as long among CARD15 variant carriers compared with other CD patients. The use of the Vienna classification, especially behaviour, in phenotypegenotype analyses has been strongly criticised by Louis et al. They determined behaviour and location of disease over 25 years of follow up from diagnosis in 297 CD patients, and found that the behaviour of CD varied dramatically over the course of the disease while location was relatively stable. ${ }^{31}$ They recommended that patients should be classified for genotype- phenotype analyses after a fixed duration of disease; this was not feasible in the present series. In our material, there was a lack of association between age at diagnosis and carrier status of the CARD15 variants. We chose to use age at diagnosis instead of age at onset as the data were collected retrospectively from patient records of the participating centres.

The CARD 15 gene product belongs to a Nod l/Apaf- 1 superfamily of apoptosis regulators and is mainly expressed in monocytes ${ }^{14}$ CARD 15 contains two $N$ terminal caspase activation recruitment domains (CARD) and several $C$ terminal leucine rich repeats (LRR) ${ }^{14}$ It is involved in the activation of the NFKB signalling pathway. ${ }^{14}$ The CARD15 $1007 \mathrm{fs}$ mutation, resulting in a truncated protein lacking part of the LRRs, is deficient in inducing LPS mediated NFKB activation. ${ }^{12}$ The $\mathrm{NF \kappa B}$ system has been shown to be activated in IBD. ${ }^{32}{ }^{33} \mathrm{Glu}-$ cocorticoids and sulphasalazine both effectively inhibit NFKB. In our material, there were no significant differences in the use of 5-ASA or steroids between carriers or non- carriers of any of the three CARDI5 variants investigated, neither were there any significant differences in the use of metronidazole or immunosuppressives between these two groups (table 3 ).

In conclusion, the allele frequencies of the three CARD15 gene variants (R702W, G908R, and 1007fs) were lower in Finns than in other populations studied to date. There was an association between the 1007fs variant allele and CD. In this retrospective study in Finnish CD patients, the NOD2 gene variants were associated with ileal disease as well as stricturing and penetrating forms of the disease, and CD patients homozygous for the CARD15 gene variants tended to suffer from a disease with early onset and a complicated course. No significant differences in the use of 5-ASA, steroids, metronidazole, or immunosuppressives were observed between CD and UC patients who were carriers of any of the three CARD15 variants investigated compared with non-carriers. There may be CARD 15 variants or genes predisposing to $C D$ among Finns other than those previously investigated in Western populations. 


\section{ACKNOWLEDGEMENTS}

We warmly thank Dr David van Heel (Oxford University, UK) for providing advice and control DNA samples for NOD2 variant analysis. Docent Markus Perola is acknowledged for advice with statistica questions. We thank the study participants and their families for volunteering for this study. We are obliged to our collaborators Professor Heikki Järvinen (Helsinki University Hospital), Dr Heimo Nurm (Turku University Hospital), Docent Anna- Liisa Karvonen (Tampere), Docent Risto Julkunen, Dr Markku Heikkinen, (Kuopio), and Docen Seppo Niemelä (Oulu). We thank Ms Tuula Soppela-Loponen for excellent technical assistance. This study was supported by a grant from the Special State Funds of the Helsinki University Central Hospital.

\section{Authors' affiliations}

T Heliö, M Lappalainen, H Fodstad, P Paavola-Sakki, U Turunen, M Färkkilä, K Kontula, Department of Medicine, Helsinki University Hospital, Helsinki, Finland

L Halme, Department of Surgery, Helsinki University Hospital, Helsinki, Finland

T Krusius, The Finnish Red Cross Blood Service, Helsinki, Finland

\section{REFERENCES}

1 Halme L, Turunen U, Heliö $T$, et al. Familial and sporadic inflammatory bowel disease: a comparison of clinical features and serological markers in a genetically homogeneous population. Scand J Gastroenterol 2002;37:692-8.

2 Hugot JP, Laurent-Puig P, Gower-Rousseau C, et al. Mapping of a susceptibility locus for Crohn's disease on chromosome 16. Nature 1996;379:821-3.

3 Ohmen JD, Yang HY, Yamamoto KK, et al. Susceptibility locus for inflammatory bowel disease on chromosome 16 has a role in Crohn's disease, but not in ulcerative colitis. Hum Mol Genet 1996;5:1679-83.

4 Parkes M, Satsangi J, Lathrop GM, et al. Susceptibility loci in inflammatory bowel disease. Lancet 1996;348:1588.

5 Brant SR, Fu Y, Fields CT, et al. American families with Crohn's disease have strong evidence for linkage to chromosome 16 but not to chromosome 12. Gastroenterology 1998;115:1056-61.

6 Cavanaugh JA, Callen DF, Wilson SR, et al. Analysis of Australian Crohn's disease pedigrees refines the localisation for susceptibility to inflammatory bowel disease (IBD-1) on chromosome 16. Ann Hum Genet 1998; 62:291-8.

7 Curran ME, Lau KF, Hampe J, et al. Genetic analysis of inflammatory bowel disease in a large European cohort supports linkage to chromosomes 12 and 1. Gastroenterology 1998:115:1066-71.

8 Hampe J, Schreiber S, Shaw SH, et al. A genomewide analysis provides evidence for novel linkages in inflammatory bowel disease in a large European cohort. Am J Hum Genet 1999;64:808-16.

9 Annese V, Latiano A, Bovio P, et al. Genetic analysis in Italian families with inflammatory bowel disease supports linkage to the IBDI locus: a GISC study. Eur J Hum Genet 1999;7:567-73.

10 The IBD International Genetics Consortium. International collaboration provides convincing linkage replication in complex disease through analysis of a large pooled data set: Crohn disease and chromosome 16. Am J Hum Genet 2001;68:1 165-71.

11 Hugot J-P, Chamaillard M, Zouali $\mathrm{H}$, et al. Association of NOD2 leucine-rich repeat variants with susceptibility to Crohn's disease. Nature 2001;411:599-603.
12 Ogura Y, Bonen D, Inohara N, et al. A frameshift mutation in NOD2 associated with susceptibility to Crohn's disease. Nature 2001;411:603-6.

13 Inohara N, Nunez G. The NOD: a signaling module that regulates apoptosis and host defense against pathogens. Oncogene 2001;20:6473-81

14 Ogura Y, Inohara N, Benito A, et al. Nod2, a Nod1/Apaf-1 family member that is restricted to monocytes and activates NF (kappa) B. J Biol Chem 2001:276:4812-18.

15 Inohara N, Ogura Y, Chen FF, et al. Human Nodl confers responsiveness to bacterial lipopolysaccharides. J Biol Chem 2001;276:2551-4.

16 Hampe J, Cuthbert A, Croucher P, et al. Association between insertion in NOD2 gene and Crohn's disease in German and British populations. Lancet 2001;357:1925-8.

17 Ahmad T, Armuzzi A, Bunce M, et al. The molecular classifiacation of the clinical manifestations of Crohn's disease. Gastroenterology 2002; 122:854-66

18 Cuthbert AP, Fisher SA, Mirza MM, et al. The contribution of NOD2 gene mutations to the risk and site of disease in inflammatory bowel disease. Gastroenterology 2002; 122:867-74.

19 Lesage S, Zouali H, Cezard JP, et al. CARD15/NOD2 mutational analysis and genotype-phenotype correlation in 612 patients with inflammatory bowel disease. Am J Hum Genet 2002:70:845-57.

20 Paavola P, Heliö T, Kiuru M, et al. Genetic analysis in Finnish families with inflammatory bowel disease supports linkage to chromosome 3 p21. Eur J Hum Genet 2001;9:328-34.

21 Lennard-Jones JE. Classification of inflammatory bowel disease. Scand J Gastroenterol 1989;24(suppl):2-6.

22 Gasche C, Scholmerich J, Brynskov J, et al. A simple classification of Crohn's disease: report of the working party for the World congresses of gastroenterology Vienna 1998. Inflamm Bowel Dis 2000;6:8-15.

23 Inoue N, Tamura K, Kinouchi Y, et al. Lack of common NOD2 variants in Japanese patients with Crohn's disease. Gastroenterology 2002;1 23:86-91

24 Kere J. Human population genetics: lessons from Finland. Annu Rev $G$ Genomics Hum Genet 2001:2:103-28.

25 Peltonen L, Jalanko A, Varilo T. Molecular genetics of the Finnish disease heritage. Hum Mol Genet 1999;8:1913-23.

26 Vuorio AF, Aalto-Setälä K, Koivisto UM, et al. Familial hypercholesterolemia in Finland: common, rare and mild mutations of the LDL receptor and their clinical consequences. Finnish FH-group. Ann Med 2001;33:410-21.

27 Piippo K, Swan $\mathrm{H}$, Pasternack $M$, et al. A founder mutation in the potassium channel KCNQ1 in long QT syndrome: implications for estimation of disease prevalence and molecular diagnostics. J Am Coll Cardiol 2001;37:562-8

28 Peltonen L, Palotie A, Lange K. Use of population isolates for mapping complex traits. Nat Genet 2000;1:182-90.

29 Eaves IA, Merriman TR, Barber RA, et al. The genetically isolated populations of Finland and Sardinia may not be a panacea for linkage disequilibrium mapping of common disease genes. Nat Genet 2000;25:246-7.

30 Hampe J, Grebe J, Nikolaus S, et al. Association of NOD2 (CARD 15) genotype with clinical course of Crohn's disease: a cohort study. Lancet 2002;359:1661-5.

31 Louis E, Collard A, Oger AF, et al. Behaviour of Crohn's disease according to the Vienna classification: changing pattern over the course of the disease. Gut 2001;49:777-82.

32 Schreiber S, Nikolaus S, Hampe J. Activation of nuclear factor kappa B in inflammatory bowel disease. Gut 1998;42:477-84.

33 Rogler G, Brand D, Vogl D, et al. Nuclear factor kappaB is activated in macrophages and epithelial cells of inflamed intestinal mucosa. Gastroenterology 1998;115:357-69. 